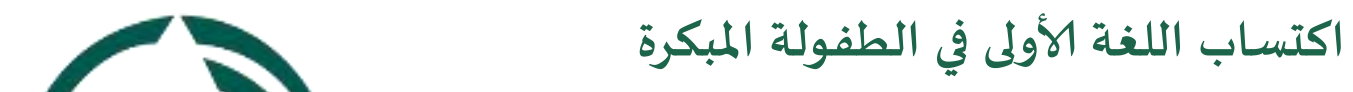

\title{
Nafiul Huda
}

\author{
Institut Pesantren KH. Abdul Chalim \\ (huda.nafiul18@gmail.com)
}

Submitted: 2019-04-11

Accepted: 2019-04-18

Approved: 2019-05-28

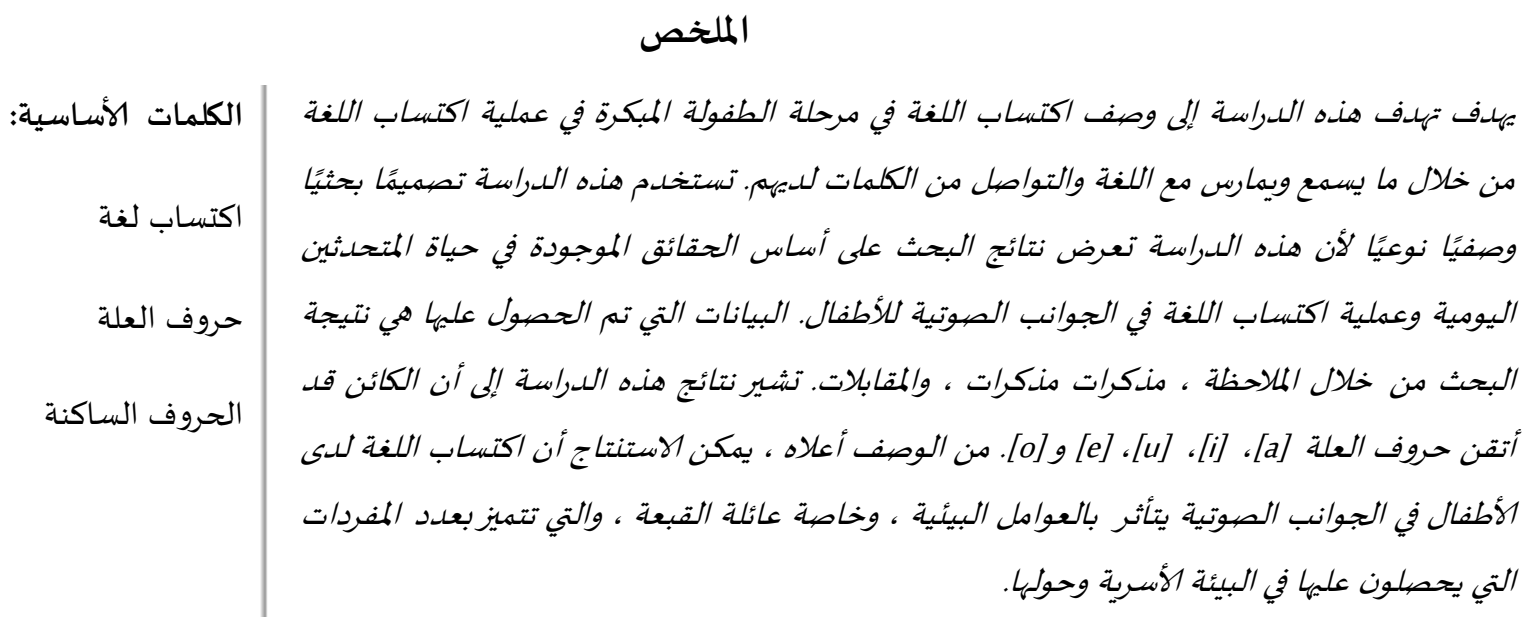

\section{Kata Kunci:}

Pemerolehan bahasa

Anak usia dini

Huruf vokal

Huruf konsonan

\section{ABSTRAK}

Pembahasan ini mempunyai tujuan ingin menjelaskan bagaimanakah fase atau tahapan perolehan bahasa pada anak usia dini dalam proses perolehan bahasanya dari apa yang didapat baik dari yang didengar maupun dipraktikkan dengan bahasa dan komunikasi dari kata-kata yang mereka punyai. Pembahasan ini menggunakan penulisan deskriptif kualitatif karena pembahasan ini menyajikan hasil pembahasan berdasarkan fakta dan data dalam kehidupan sehari-hari para penutur dan proses penguasaan bahasa dalam aspek vokal anak usia dini. Data yang diperoleh adalah hasil pembahasan dari cara literasi, observasi, dan diskusi. Hasil pembahasan ini memberi tahukan bahwa objek dalam hal ini adalah anak usia dini telah menguasai vokal [a], [i], [u], [e] dan [o]. Menurut uraian tersebut, dapat diketahui bahwa perolehan bahasa pada anak usia dini dalam aspek vokal dipengaruhi oleh faktor lingkungan, terutama keluarga. Hal ini ditandai dengan jumlah kata yang didapati dari dalam dan sekitar lingkungan keluarga.
Nafiul Huda

اكتساب اللغة الأولى في الطفولة المبكرة

\footnotetext{
http://e-journal.ikhac.ac.id/index.php/alsuna https://doi.org/10.31538/alsuna
} 
اللغة هي أداة اتصال حصل عليها البشر منذ الولادة. يبدأ إتقان الطفل للغة من خلال اكتساب اللغة الأولى والتي غالباً ما تسهى اللغة الأم. اللغة هي في الأساس نظام رمز صوت تعسفي يستخدما أعضاء المجموعات الاجتماعية للعمل معًا والتواصل وتحديد هويتهم. علاوة على ذلك ، أوضح أوين أنه يمكن تعريف اللغة على أنها رمز مقبول اجتماعيًا أو نظام تقليدي لنقل المفاهيم من خلال استخدام الرموز المطلوبة ومجموعات الرموز التي تحكمها الأحكام اكتساب اللغة وفقًا لـ (Maksan:1993) هي عملية إجادة للغة يقوم بها شخص بدون وعي ، ضمنيًا وغير رسمي. ينص على أن اكتساب اللغة هو عملية طبيعية لإتقان اللغة التي يؤديها الأطفال عندما يتعلم لغته. الشخص ليس لدياه فجأة قواعد كاملة مع جميع القواعد في دماغه. يتم الحصول على اللغة الأولى من قبل الطفل في عدة مراحل ، وكل مرحلة تالية أقرب إلى قواعد اللغة الخاصة بالبالغين. مصطلح الاستحواذ هو ما يعادل اكتساب الكلمة. يستخدم هذا المصطلح في عملية إتقان اللغة الأولى باعتبارها واحدة من التطورات التي تحدث في إنسان منذ الولادة. بطبيعة الحال ، سيتعرف الأطفال على اللغة كوسيلة للتواصل مع الأشخاص من حولهم. تسمى اللغة الأولى التي يعرفها الطفل ويتقها لاحقًا اللغة الأم. يرتبط اكتساب اللغة الأولى ارتباطًا وثيقًا بالتطور الاجتماعي للأطفال وتشكيل الهوية الاجتماعية. تعلم اللغة الأولى هو أحد التطورات الشاملة للطفل كونه عضوًا في مجتمع (Yogotama:2011). فيما يتعلق اكتساب اللغة أو اكتساب اللغة هناك العديد من المعاني. وفقًا لتاريجان (تاريجان ، 1911) ، فإن اكتساب اللغة هو عملية امتلاك مهارات لغوية ، كلاهما في شكل فهم أو الكشف الطبيعي ، دون الذهاب إلى أنشطة التعلم الرسمي. علاوة على ذلك ، ينص على أن اكتساب اللغة هو عملية إتقان اللغة التي يتم إجراؤها بشكل طبيعي من قبل الأطفال عندما يتعلم الأطفال لغتهم الأم. وفقًا لما ذكره مانورونج ، فإن اكتساب اللغة للأطفال له خصائص مستمرة ، وهو عبارة عن سلسلة من الوحدة ، ويبدأ من كلام بسيط مكون من كلمة واحدة لتحقيق مزيج أكثر تعقيدًا من الكلمات والجمل (Salnita:2019).عادة ما يتم تمييز اكتساب اللغة عن تعلم اللغة. يحدث اكتساب اللغة عادة بشكل طبيعي ، عن غير قصد ، يتم الحصول عليه في نطاق الحياة اليومية ، بينما في تعلم اللغة ، يتم الحصول على اللغة بعد دراستها بشكل رسمي مع الامتثال للقواعد النحوية المعمول بها(Chaer:2003) .وفقًا لترويكي في الحصول على لغة ثانية ، يفترض أن جميع الميزات المهمة المفترضة كلغة 
ثانية هي لغات في مرحلة الطفولة المبكرة. عادةً ما يبدأ اكتساب اللغة الثانية من سن الثالثة ويتعلمون لغة الأشخاص المحيطين بهم (Troike:2006). منذ سن مبكرة ، تفاعل الأطفال في بيئتهم الاجتماعية. غالبًا ما تمنح الأم فرصة للأطفال للمشاركة في التواصل الاجتماعي ، لذلك عندما يعرف الطفل أولاً أن هذا العالم هو مكان يشارك فيه الناس مشاعرهم. من خلال اللغة الأولى (B1) ، يتعلم الطفل أن يكون عضواً في المجتمع B1 .هي إحدى وسائل التعبير عن المشاعر والرغبات والقناعات ، بأشكال اللغة التي تعتبر موجودة. يتعلم الأطفال أيضًا أن هناك أشكالًا غير مقبولة لأفراد مجتمعهم ، ويجب ألا يعبر الأطفال دائمًا عن مشاعرهم بصراحة. اكتساب اللغة الأولى أو اللغة الأم للأطفال حول العالم هي نفسها. لا يتسبب تشابه عملية الاستحواذ فقط في عناصر البيولوجيا وعلم الأعصاب ، ولكن أيضًا في جانب عقلية اللغة(Arifudin:2010).لذلك ، يمكن أن نستنتج أن اللغة الأولى أو اللغة الأم هي اللغة التي حصل عليها الفرد أولاً في حياته. ستكون هذه اللغة هي اللغة الأكثر تهديدًا وغالبًا ما يتم استخدامها من قبل مستخدمي اللغة. تم إجراء بحث عن اللغة الأولى للأطفال في إندونيسيا. من خلال البحث الذي أجراه ، يمكن استنتاج أنه من خلال تعبيرات الوجاه والإيماءات التي يظهرها الطفل يمكن أن تساعد الأشخاص من إنماء حولهم على فهم اللغة التي يتحدثها الطفل. حصل البحث الذي أجراه على نتائج أنها في عمر ثلاث سنوات لم يتمكن الطفل من نطق الصوتيات / / / / / / بشكل صحيح. الكلمات التي يتقها الأطفال هي كلمات قريبة من بيئة الطفل وجميع الكلمات المنطوية على أطفال في هذا العمر تحتوي على معنى تدويني. وقد توصل البحث الذي أجراه إلى النتائج أنه في سن الرابعة من العمر ، كان الأطفال في البناء البسيط قادرين على نطق الجمل في جمل توضيحية واستجوابية وضرورية. وفقًا لـ Ingram في دراسة، تم تقسيم اكتساب اللغة الأولى إلى أربع فترات. أولاً ، تتميز المرحلة


(1-7.7 سنة) على عدد من الأصوات ذات المعاني الخاصة التي تعبر عن فكرة الجملة ككل ، ولكن لا يوجد دليل على أن الأطفال يفهمون القواعد. ثالثًا ، تدرك الفترة الثانية (7. (-..ب) من الأطفال أن كل شيء له


تكوين جمل جيدة بمعنى احتواء الكلمات على العلاقات النحوية الرئيسية للموضوع والمسند.

Nafiul Huda

اكتساب اللغة الأولى في الطفولة المبكرة
(61) 
يمكن تقسيم تطور اكتساب اللغة للأطفال إلى ثلاثة أجزاء ، على النحو التالي. أولا ، تطوير ما

قبل المدرسة. تتكون مرحلة التطوير ما قبل المدرسة من ثلاث مراحل من التطور ، وهي تطوير علم اللغة ، مرحلة كلمة واحدة ، والكلام في بداية المجموعة. في النمو اللغوي للأطفال ، يطور الأطفال مفهومهم الذاتي. حاول أن يميز نفسـه عن الموضهوع ، نفسـه مع الآخرين ، والعلاقات مع الأشياء والأفعال. في المرحلة الأولى ، يواصل الطفل محاولة جمع أسماء الأشياء والأشخاص الذين يقابلونهم. ثانيا ، تطوير الكلام التوافقي. الثالثة ، وتطوير الفترة المدرسية. من المتوقع أن يكون هذا البحث مفيدًا من الناحية النظرية والعملية. من الناحية النظرية ، قد يكون من المفيد زيادة المعرفة حول اكتساب الأطفال للغة الأولى. يمكن أن يكون هذا البحث مفيدًا للآباء ليكونوا قادرين على فهم ما يقوله طفلهم. إذا تمكن الوالدان من فهم ما يقوله الطفل ، فسيكون الطفل

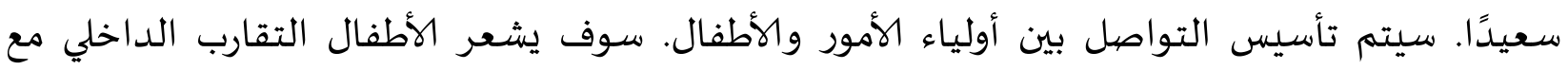
الآباء. هذا سوف يساعد في علم نفس الأطفال ينمو بشكل أفضل.

\section{منهجية البحث}

الطريقة المستخدمة في هذا البحث هي طريقة وصفية ذات منهج نوعي. وفقًا، فإن الطريقة الوصفية هي طريقة لفحص مجموعة من الأشخاص ، أو الموضوع ، أو التفكير في الوقت الحاضر. يتم استخدام هذه الطريقة الوصفية لإعطاء نظرة عامة على نتائج جمع البيانات التي أجراها الباحثون ، من خلال المقابلات (الأباء ، الأطفال) والملاحظات المباشرة إلى الميدان ، حول الألفاظ التي ينطق بها موضوعات البحث. تم اختيار الأساليب الوصفية من قبل الباحثين لأن هذه الطريقة يمكن أن توفر صورة دقيقة قدر الإمكان عن الأفراد أو الظروف أو اللغات أو الأعراض أو المجموعات. وقد أجري هذا البحث من خلال مراقبة مباشرة الأطفال الذين كانوا موضوع الدراسة. لأن الطفل ليس لديا فجأة قواعد منتظمة في دماغه. تتعلق المرحلة الأولى من اكتساب اللغة بتطوير لغة الأطفال. هذا لأن اللغة الأولى يحصل عليها شخص ما عندما يكون طفلاً. طبق هذا الباحث تقنيات الاستماع الماهرة. استمع الباحث إلى الكلمات التي يمكن أن ينطق بها الطفل بعد ذلك. تفاعل الباحث أيضًا وشجع موضوعات البحث على قول الكلمات. يتم جمع البيانات من خلال مراقبة وفهم اللغة التي يتحدث بها موضوع البحث. يسجل الباحث كل كلمة وكلام ينطق به موضوع البحث. أجريت هذه الدراسة لمدة r أسابيع تقريبًا.

Nafiul Huda

اكتساب اللغة الأولى في الطفولة المبكرة 
تتكون مرحلة تحليل البيانات في هذه الدراسة من ثلاث مراحل ، وهي على النحو التالي. أولا ، الحد. يتم التخفيض بأربعة طرق ، هي: أ) نسخ بيانات الكلام في شكل مكتوب ؛ ب) يتم بعد ذلك تحديد البيانات التي تم نسخها ؛ ج) ينفذ عملية التصنيف ؛ د) تفسير البيانات المصنفة سابقا. الثانية ، مرحلة العرض. في هذه المرحلة من العرض ، يتم تقديم البيانات المصنفة في شكل جدول. الثالثة ، مرحلة استخلاص النتائج. في هذه المرحلة الثالثة ، يتم تنفيذ عملية إعادة التحقق من البيانات الأولية التي تم جمعها (مايلز وهوبرمان ، ع ا. (Y).

\section{المباحث ونتائجها}

الكلمات الأولى التي يحصل عليها في هذه المرحلة هي عادة كلمات تعبر عن أفعال وخصائص وأسماء. إن تطوير اللغة الأولى للأطفال أسهل في تحديد الكلمات من الكلمات المنطوقة. عدد الكلمات التي يتحدثها الطفل هو مؤشر أو دليل لتطوير اللغة.

الكلمات المنطوقة والمتقنة من قبل الأطفال هي الأسماء والأفعال والصفات القريبة من بيئتهم

اليومياة. على الرغم من أن المقطع الأخير فقط هو المنطوق ، إلا أنه له معنى يمكن فهماه من قبل آبائهم. الطفل ليس لديا فجأة قواعد منتظمة في دماغه. تتعلق المرحلة الأولى من اكتساب اللغة بتطوير لغة الأطفال. وذلك لأن اللغة الأولى يحصل عليها شخص ما عندما يكون طفلاً. صوتيًا ، الأطفال حديثي


فقط من حجم الشخص البالغ. يتم ملء تجويف الفم الضيق تقريبا مع اللسان. زيادة العمر سوف يوسع تجويف الفم. يوفر هذا النمو مساحة أكبر للأطفال لإنتاج أصوات اللغة.

في هذه المرحلة ، فإن أصوات اللغة التي ينتجها الطفل ليست ذات معنى بعد. تشبه الأصوات بالفعل بعض حروف العلة أو الحروف الساكنة. لكن ، بشكل عام ، لا يشير الصوت إلى بعض الكلمات والمعاني. لتوضيح المزيد من مراحل اكتساب اللغة الأولى ، فيما يلي وصف لمراحل اكتساب لغة الطفل.

المرحلة الأولى ،تستمر هذه المرحلة من الطفل المولود حتى عمر rا شهرًا. أ ) في عمر .-ب أشهر ،

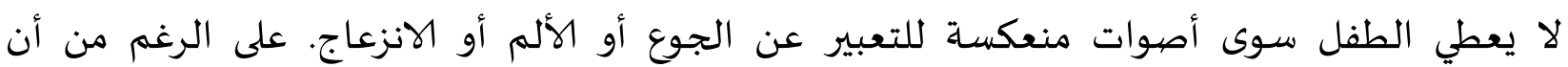


الأصوات لا تعني اللغة ، إلا أن الأصوات هي المادة الخاصة بالكلمة التالية. ب) في عمر ؟-ه أشهر ، يبدأ الطفل في إصدار الأصهوات الصوتية التي تمتزج مع الأصهوات الشبيهة بالأصهوات. يظهر هذا الصوت عادةً كرد فعل على ابتسامة أم خطاب والدته أو أي شخص آخر. ج) في عمر ع-V أشهر ، يبدأ الطفل في أن يبدو سليمًا لمدة أطول. الأصوات المتشابهة أو الصوتية الشبيهة أكثر تنوعًا. د) في سن ج-T ا شهرًا ، يبدأ الأطفال في الدردشة. الثرثرة هو نفس تكرار الحروف الساكنة.

المرحلة كلمة واحدة، تحدث هذه المرحلة عندما يكون عمر الأطفال ب ا-1 شهرًا. في هذا الوقت ،

يستخدم الأطفال كلمة واحدة لها معنى يمثل الفكرة بأكملها. بالمعنى الدقيق للكلمة ، تمثل كلمة واحدة جملة أو جمل أو أكثر. لذلك ، تسمى هذه العبارة أيضًا مرحلة هولوفراسيس. موضوع البحث في مرحلة


حول موضيوع البحث.

المرحلة كلمات ثانية، تحدث هذه المرحلة عندما يكون عمر الأطفال ما بين \\ إلى ع شهرًا. في

هذا الوقت ، تتطور المفردات والقواعد للأطفال بسرعة. يبدأ الأطفال باستخدام كلمتين في الكلام. بدأ حديثه ليكون التلغرافي. بمعنى أن ما يقوله الأطفال هو مجرد كلمات مهمة ، مثل الأسماء والصفات والأفعال. تم حذف الكلمات غير المهمة ، كما لو كنا نكتب برقية.

المرحلة دمج الكلمات، تحدث هذه المرحلة عندما يكون عمر الأطفال ب-ه سنوات أو حتى بدء الدراسة. في سن ب-ع سنوات ، يبدأ خطاب الأطفال في أن يكون أطول وأن القواعد أكثر انتظامًا. لم يعد يستخدم كلمتين فقط ، بل ثلاث كلمات أو أكثر. في سن 0-7 سنوات ، تشباه لغة الأطفال لغة البالغين.

الخاتمة

بناءً على نتائج هذه الدراسات ، يمكن استنتاج أن اكتساب اللغة هو العمليات التي تنطبق على

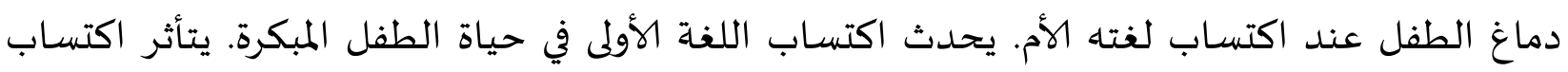
اللغة الأولى للطفل بشدة بالبيئة الأسرية المحيطة بالطفل. من حيث القيم الاجتماعية والثقافية التي يتم الالتزام بها سيتم استيعابها في العقل الباطن للإنسان. ثم ، بمرور الوقت ونمو الطفل ، سيحصل الطفل

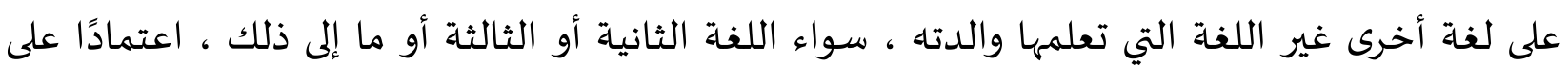


البيئة الاجتماعية والمستوى المعرفي الذي يمتلكه الطفل من خلال عملية التعلم. تتعامل اللغة الأولى مع اكتساب اللغة ، بينما تتعامل في اللغة الثانية مع تعلم اللغة.

ALSUNA Vol. 2 (1), 2019 


\section{Bibliography}

Arsanti Meilan. "Pemerolehan bahasa pada anak (kajian psikolinguistik)". Jurnal PBSI, vol. 3, no. 2,2014 .

Chaer, Abdul. Psikolinguistik: Kajian Teoritik. PT Rineka Cipta, 2003.

Dardjowidjojo, Soenjono. Kisah Pemerolehan BahasaAnak (Echa). Unika Atma Jaya, 2000.

Dardjowidjojo. Psikolinguistik. Pengantar Pemahaman Bahasa Manusia. Yayasan Obor Indonesia, 2003.

Ellis, Rod. Understanding Second Language Acquisition. Oxford University Press, 1986.

Manaf. Sintaksis: teori dan terapannya dalam bahasa Indonesia. Sukabina Press, 2010.

Moleong, Lexy. Metodologi Pembahasan Kualitatif. Remaja Rosdakarya Offset, 2005.

Nazir. Metode Pembahasan. Ghalia Indonesia, 1988.

Rafiek, Muhammad. Psikolinguistik: Kajian Bahasa Anak dan Gangguan Berbahasa. UM Press, 2010.

Tarigan, Henry Guntur. Pengajaran Pemerolehan Bahasa. Angkasa, 1988.

Nafiul Huda اكتساب اللغة الأولى في الطفولة المبكرة
ALSUNA Vol. 2 (1), 2019 